\title{
Omitting the First Step of GLIM Framework Yielded Better Accordance With PG-SGA in Chinese Ambulatory Cancer Patients: a Prospective Cross- sectional Study
}

Yanfei Wang

peking university cancer hospital

Ziqi Liu

Peking University Cancer Hospital

yunyi Wang

Peking University Cancer Hospital: Beijing Cancer Hospital

Xiaoyan Chen

Peking university cancer hospital

\section{Zhongfen Liu}

Peking university cancer hospital

\section{Yanqun Zheng}

Peking University Cancer Hospital: Beijing Cancer Hospital

\section{Danfeng Jiang}

Peking University Cancer Hospital: Beijing Cancer Hospital

\section{Lina Xia}

Peking University Cancer Hospital: Beijing Cancer Hospital

\section{Yu Fang}

Peking University Cancer Hospital: Beijing Cancer Hospital

\section{Zhi Peng}

Peking University Cancer Hospital: Beijing Cancer Hospital

Wei Liu ( $\boldsymbol{\sim}$ beijingzlliuwei@163.com )

Peking university Cancer Hospital https://orcid.org/0000-0002-1534-1823

\section{Research Article}

Keywords: ambulatory cancer patients, GLIM, PG-SGA, MST, MUST, NRS2002

Posted Date: November 29th, 2021

DOI: https://doi.org/10.21203/rs.3.rs-1013336/v1

License: () (1) This work is licensed under a Creative Commons Attribution 4.0 International License. Read Full License 
Page 2/17 


\section{Abstract}

Background and Aims: The Global Leadership Initiative on Malnutrition (GLIM) criteria is a new framework for diagnosing malnutrition in combination of phenotypic and etiologic criteria after nutrition screening using validated screening tools. The aim of this study was to evaluate the efficacy of malnutrition screening tool (MST), malnutrition universal screening tool (MUST) and nutritional risk screening 2002 (NRS2002) as the first step of GLIM framework in comparison to Patients-Generated Subjective Global Assessment (PG-SGA) in Chinese ambulatory cancer patients.

Methods: A single-center prospective cross-sectional study was conducted. Nutritional screening and assessment were performed within $4 \mathrm{~h}$ after admission to the hospital using a structured questionnaire including MST, MUST, NRS2002, PG-SGA and GLIM, with supplement information of calf circumference (CC) measurement and body composition measurement using bioelectrical impedance analysis (BIA). Malnutrition diagnosis made by GLIM framework using MST, MUST or NRS2002 as the first step or without screening step were compared to PG-SGA separately. Sensitivity, specificity, positive (PPV) and negative (NPV) predictive values and $\mathrm{K}$ values were used to evaluate performance of the screening tools.

Results: Of the 562 included patients, Of the participants 62.8\% (355/562) were male and 37.2\% (210/562) were female, with a male to female radio of 1.69:1. The median age of the patients was 59.0 years (range, 21$82 \mathrm{y}$; interquels range $52.0-65.0 \mathrm{y}$ ). From the 562 patients included in the study, $41.8 \%$ of patients were evaluated as malnutrition ( $P G-S G A \geq 4$ ) and $11.9 \%$ were diagnosed as severe malnutrition (PG-SGA D). For GLIM criteria, omitting the screening step yielded fair accordance with PG-SGA in diagnosing malnutrition $(\mathrm{K}=0.623)$ and severe malnutrition ( $\mathrm{k}=0.515)$. Using MUST as the first step of GLIM framework has better performance $(\mathrm{K}=0.614 ; \mathrm{K}=0.515)$ than using MST $(\mathrm{K}=0.504, \mathrm{~K}=0.496)$ or NRS2002 $(\mathrm{K}=0.363, \mathrm{~K}=0.503)$ as the screening tool regardless of severity gradings.

Conclusions: Using PG-SGA as the standard, GLIM framework omitting first step has better performance compared with using MST, MUST or NRS2002 as the screening tool. Among the screening tools validated to be used in the first step of GLIM framework, MUST may be the better choice for ambulatory cancer patients.

\section{Introduction}

Cancer patients are at high risk of malnutrition. It is estimated that around $30-90 \%$ of cancer patients suffer from malnutrition due to either physical and metabolic effects of the cancer or the adverse effects of anticancer treatments $[1,2]$. Malnutrition is associated with reduced treatment effectiveness $[3,4]$, increased treatment toxicities[5], impaired functional status and quality of life[6], higher health care cost[7] and poorer survival[8-10]. Considering the high incidence and the multiple adverse impacts of malnutrition on cancer patients, routine nutritional screening and assessment are suggested in different clinical settings.

The Global Leadership Initiative on Malnutrition (GLIM) operational diagnostic criteria were developed from 2016 to 2018 by several global clinical nutrition societies in order to standardize the practice of malnutrition diagnosis in different clinical settings worldwide[11, 12]. The first step is to evaluate the nutrition status using any validated malnutrition screening tool[11] to identify patients at risk of malnutrition followed by a second 
step of assessment for diagnosis of malnutrition and severity grading. Which screening tool is more appropriate in different clinical setting is still unknown.

Since its publication in 2019, there have been emerging studies assessing the accordance between previous nutrition screening or nutrition evaluation tools and the validity of GLIM framework for diagnosing malnutrition. One of the major issues in validating GLIM criteria is to choose a proper standard as the comparator. As suggested by a recently published guidance review on validation of GLIM framework, standardized tools which had been validated such as the Subjective Global Assessment (SGA), the Patient-generated Subjective Global Assessment (PG-SGA) or Minimal nutrition Assessment (MNA) could be used as comparator for determining validity of GLIM[13, 14]. PG-SGA is a nutrition assessment tool adapted for oncology patients from SGA[15], and have been commonly used among oncology patients worldwide. Therefore, in this study we chose PG-SGA as the comparator to evaluate the performance of GLIM using different definitions of criteria.

Due to the increasing cancer incidence and the progression achieved in cancer treatment, the number of cancer patients increases dramatically, and many cancers may be converted to chronic diseases[4]. More and more cancer patients are treated in day care settings. In day care setting, the turnover rates of day care centers are often high, excessive complexity of assessment methods can lead to inadequate screening. As such, it's important to figure out the most effective and efficient tools for nutrition screening and assessment in order to facilitate timely nutrition care for cancer patients [16].

The current study aimed to evaluate the diagnostic capacities of three frequently used malnutrition screening tools including malnutrition screening tool (MST), malnutrition universal screening tool (MUST) and nutritional risk screening 2002 (NRS2002) as the first step of GLIM framework in light of the consistency between GLIM and PG-SGA in Chinese ambulatory adult cancer patients.

\section{Materials And Methods 2.1. Study design}

Our single-center cross-sectional, observational study was conducted at the Day Oncology Unit in Peking University Cancer Hospital, Beijing, China, spanning 3 weeks from 11 November 2020 to 10 December 2020.

\subsection{Participants}

The eligibility criteria included: (1) diagnosed with cancer by pathology, (2) age $\geq 18 y$, (3) receiving in-chair intravenous treatment, and (4) having normal cognitive function. Patients were excluded from the study if they were: (1) in poor performance status, namely Eastern Cooperative Oncology Group (ECOG) score >2; (2) unable to stand up due to illness, or (3) unwilling to participate in the study.

\subsection{Data collection}

To simplify data collection, we transferred all items of the MST[17], MUST[18], NRS2002[19], CLIM criteria[11, 12] and PG-SGA[15] into a structured questionnaire with different assessment categories in the research design phase. 
Before the data collection, three doctors and one nutritionist were trained by the same instructor. The training session began with the content and standard procedures of the data collection, followed by a practice session. Any confusion and discrepancies were discussed and solved during the session. The trained doctors and nutritionist completed the data collection by applying a structured questionnaire within $4 \mathrm{~h}$ of hospital admission.

The structured questionnaire included all items from the MST, MUST, NRS2002, PG-SGA and GLIM. Decreased food intake was asked for the past week, 2 weeks and 1 month based on patients' estimation of reduction in general food intake. Body weights in the past $(0.5,1,2,3,6$ months and over 6 months separately) were recorded based on patients' self-reporting and electronic medical record (if available), and then the percentage of unintentional weight loss was calculated.

The collected data also included patients' general information, plasma C-reactive protein (CRP) values, anthropometric measurement, and body composition measurement.

Patients' general information, including age, sex, tumor site, tumor stage, comorbidities, and treatment, was obtained from medical records.

CRP were tested using fasting blood, and the values within 1 week of admission were obtained from electrical medical record (if available).

The anthropometric measurement included body weight, height, body mass index (BMI), percentage of weight loss, and calf circumference (CC). The patient's body weight and height were measured in light indoor clothing without shoes at the time of admission. BMI was calculated as the weight $(\mathrm{kg}) / \mathrm{height}(\mathrm{m})^{2}$. The CC of both sides were measured using a flexible and non-elastic tape, and the smaller value was recorded for use.

All patients underwent human body composition measurement by bioelectrical impedance analysis (BIA) using InBody770 (InBody Co., Ltd., Cheonan, Korea) upon entering the ward before receiving intravenous treatment. Inbody770 reports FFMI and appendicular skeletal muscle index (ASMI) values.

After the data collection, researchers calculated the summary scores for the MST, MUST, NRS2002 and PG-SGA, and compared the summary scores against the GLIM criteria. Nutritional diagnoses made by GLIM criteria followed by MST, MUST and NRS2002 were compared with diagnoses made by PG-SGA respectively in order to evaluate the capacity of different nutrition screening tools.

\subsection{PG-SGA}

Nutritional status was assessed with the PG-SGA. PG-SGA consists of two sections, namely patient- and clinician-completed components. The patient-completed component includes four aspects: weight loss, nutritional impact symptoms, food intake, and functional capacity. The clinician-completed component assesses four aspects: disease situation, age, metabolic stress, and physical examination. Based on the above assessments, patients were classified as well-nourished (PG-SGA: A, score 0-1), suspected of being or moderately malnourished (PG-SGA: B, score 2-8), or severely malnourished (PG-SGA: C, score 29)[15]. In order to guide clinical practice, category $B$ can be further divided into suspicious or mild malnutrition (score 2-3) and moderate malnutrition (score 4-8); the latter requires nutritional intervention and symptomatic treatment[20]. In 
this study, patients with PG-SGA score $\geq 4$ were diagnosed with malnutrition, and those with PG-SGA score $\geq 9$ (i.e., category C) were identified as having severe malnutrition.

\subsection{MST}

The MST consisted of two questions regarding appetite and recent unintentional weight loss. Patients with MST score of $\geq 2$ were at a risk of malnutrition[17].

\subsection{MUST}

The MUST takes into account parameters including BMI, unintentional weight loss, and acute disease comprising nutritional intake for $>5 \mathrm{~d}$, and MUST scores are rated as descripted in literature, with a score of 0 indicating low risk, 1 medium risk, and 2 high risk[18]. In this study, patients with MUST moderate or high risk were considered as at malnutrition risk and further evaluated by GLIM criteria.

\subsection{NRS2002}

For the NRS2002, parameters took into account including the severity of disease, impaired nutritional status, and the age. Impaired nutritional status included unintentional weight loss, reduced food intake, and low BMI. A final score of the NRS2002 $\geq 3$ denoting a high nutritional risk[19].

\subsection{GLIM criteria}

The interpretation of GLIM criteria was descripted in a previously published article assessing the value of reduced muscle mass evaluation[21] as follows.

\subsubsection{Step 1: Nutrition screening}

To evaluate the capacity of the screening tools in the first step of GLIM framework, the MST, MUST and NRS2002 were used to screen malnutrition risk separately followed by malnutrition diagnosing. Patients evaluated as at risk of malnutrition by the MST, MUST and NRS2002 were further assessed by step 2 and step3 to made nutritional diagnosis respectively. Nutritional diagnosis was also made omitting the malnutrition screening step.

\subsubsection{Step 2: Assessment for diagnosis}

The GLIM criteria consist of three phenotypic (weight loss, low BMI, and reduced muscle mass) and two etiologic (reduced food intake/assimilation and disease burden/inflammation) criteria.

Phenotypic Criteria

In this study, phenotypic criteria were defined as follows:

A. Weight loss: weight loss is defined as unintentional weight loss of $>5 \%$ within the past 6 months or $>10 \%$ beyond 6 months.

B. Low BMI: a low BMI for Asians is considered when $\mathrm{BMI}<18.5 \mathrm{~kg} / \mathrm{m}^{2}$ if age $<70$ y or $\mathrm{BMI}<20 \mathrm{~kg} / \mathrm{m}^{2}$ if age $>70 \mathrm{y}$. 
C. Reduced muscle mass: Reduced muscle mass is identified if the patients meet either one of the following three criteria: (1) $\mathrm{CC}<34 \mathrm{~cm}$ in men or CC $<33 \mathrm{~cm}$ for women[22]. (2) $\mathrm{FFMl}<17 \mathrm{~kg} / \mathrm{m} 2$ in men or $<15 \mathrm{~kg} / \mathrm{m} 2 \mathrm{in}$ women, as established by the European Society for Clinical Nutrition and Metabolism (ESPEN)[23]. (3) ASMI $<7.0 \mathrm{~kg} / \mathrm{m} 2$ in men or $<5.7 \mathrm{~kg} / \mathrm{m} 2$ in women according to the Asian Working Group for Sarcopenia (AWGS)[22].

\section{Etiologic Criteria}

There are two different etiologic criteria in GLIM criteria. In this study, each etiologic criterion was defined as follows:

A. Reduced intake or assimilation: reduced intake or assimilation is defined as intake $\leq 50 \%$ of energy requirement for $>1$ week, or reduction for $>2$ weeks, or the presence of disorders which affect assimilation, or gastrointestinal symptoms which were shown in the PG-SGA questionnaire.

B. Inflammation: inflammation is identified by plasma C-reactive protein (CRP) $>8 \mathrm{mg} / \mathrm{L}$ (if available), or current diseases/injury with which inflammation is likely to be associated with $[11,12]$ according to medical records. Since all participants of this study were with malignant disease, malignancy was not used as an indicator for inflammation.

Diagnosis of Malnutrition

When a patient met at least one phenotypic criterion and at least one etiologic criterion, the patients would be diagnosed as malnutrition by GLIM criteria in this study.

\subsubsection{Step 3: Severity grading}

After the diagnosis of malnutrition, the severity of malnutrition is identified solely by phenotypic criteria. For unintentional weight loss, the cut-off values to grade malnutrition severity were weight loss of $>10 \%$ within the past 6 months or $>20 \%$ beyond 6 months as presented in criteria[11, 12]. For low BMI, The cut-off values to grade malnutrition severity were $\mathrm{BMl}<17.0 \mathrm{~kg} / \mathrm{m} 2$ if age $<70$ y or $\mathrm{BMl}<17.8 \mathrm{~kg} / \mathrm{m} 2$ if age $>70 \mathrm{y}$ according to a recent study conducted in Japanese population[24]. Since there is no consensus on the cut-off value in grading severity of muscle mass reduction by anthropometric measurements referring to $\mathrm{CC}$, or FFMI and ASMI acquired by BIA, muscle mass reduction was not further divided into different severity grades.

\subsection{Ethics statement}

The study was approved by the independent institutional ethics committee of the Peking University Cancer Hospital. All enrolled participants signed informed consent for the scientific use of their data.

\subsection{Statistical Analysis}

All analyses were performed using the Statistical Package for the Social Sciences (SPSS), version 20.0 (IBM, Armonk, NY). Results were considered statistically significant when the $p$-value $\leq 0.05$.

Continuous variables following normal distribution were presented as mean values and standard deviations and otherwise presented as medians and quartiles. Categorical variables were presented as counts and portions. 
Statistical evaluations of the CLIM criteria compared to PG-SGA were performed. Sensitivity (SE), specificity $(\mathrm{SP})$, positive predictive value (PPV), and negative predictive value (NPV) were calculated to determine the performance of different combinations of GLIM criteria in light of PG-SGA as the gold standard. Data were expressed as a percent and 95\% confidence interval (CI). Validity statistics were calculated using all possible combinations of the GLIM phenotypic and etiologic criteria; each combination of two criteria, since in some clinical situation, not all data for each criterion were accessible. Comparisons were made to the criterion of malnutrition (PG-SGA score $\geq 4$ ) or severe malnutrition (PG-SGA score $\geq 9$ ). The agreement among the GLIM and PG-SGA was addressed by $\mathrm{k}$. $\mathrm{k}$ that is $>0.80$ is substantial, whereas $0.61-0.80$ is moderate, the presence of lower $\mathrm{K}$ values brings into question the reliability of the GLIM criteria[13,25]. The rating of validity test statistics also followed recommended cut points for sensitivity and specificity: both SE and SP > $80 \%$ is 'good'; SE or SP $>80 \%$ and both $>50 \%$ is 'fair'; SE or SP $>50 \%$ is 'poor' $[26,27]$.

\section{Results}

\subsection{Characteristics of participants}

A total of 686 patients were admitted to day oncology from 11 November 2020 to 10 December 2020, and 562 cancer patients participated in this study (see flow chart, Figure 1).

Of the participants, $62.8 \%$ (355/562) were male, and $37.2 \%(210 / 562)$ were female, with a male to female radio of 1.69:1. The median age of the patients was 59.0 years (range, 21-82y; interquartile range 52.0-65.0y). The median BMI was $22.8 \mathrm{~kg} / \mathrm{m}^{2}$ (range, $14.6-34.5 \mathrm{~kg} / \mathrm{m}^{2}$; the interquartile range was $20.5-25.2 \mathrm{~kg} / \mathrm{m}^{2}$ ). The characteristics of the patients are displayed in Table 1. 
Table 1

Characteristics of 562 patients with cancer enrolled in this study.

\begin{tabular}{|ll|}
\hline Parameter & Total $\mathbf{n = 5 6 2}$ \\
\hline Age, y (median, interquartile range) & $59(52-65)$ \\
\hline Sex, $\mathrm{n}(\%)$ & \\
\hline Male & $354(70.3)$ \\
\hline Female & $208(37.0)$ \\
\hline Tumor site, $\mathrm{n}(\%)$ & \\
\hline Upper Gastrointestinal tract & $134(23.8)$ \\
\hline Colorectal & $291(51.8)$ \\
\hline Head and neck & $1(0.2)$ \\
\hline Lung and mediastinum & $43(7.7)$ \\
\hline Breast & $21(3.7)$ \\
\hline Urogenital & $11(2.0)$ \\
\hline Gynaecology & $1(0.2)$ \\
\hline Liver,pancreas & $40(7.1)$ \\
\hline Lymphoma & $4(0.7)$ \\
\hline Other & $16(2.8)$ \\
\hline Tumor stage, $\mathrm{n} \%)$ & $10(1.8)$ \\
\hline I & $45(8.0)$ \\
\hline II & $176(31.3)$ \\
\hline III & $331(58.9)$ \\
\hline
\end{tabular}

\subsection{Statistical evaluation of nutrition screening / assessment tools compared with GLIM criteria}

27.2\% (153/562) and 59.8\% (336/562) of patients were classified as at risk of malnutrition by MST and MUST, while $24.2 \%(136 / 562)$ of the patients were classified as at nutritional risk by NRS2002.

As for nutrition assessment tools, the prevalence of malnutrition as per scored PG-SGA $\geq 4$ was $41.8 \%$ (235/562) and from which, severe malnutrition (scored PG-SGA $\geq 9$, or PG-SGA C) was 11.9\% (67/562), while 
the prevalence of malnutrition and severe malnutrition diagnosed based on GLIM criteria without the first step or using different screening tools were listed in Table 2.

Table 2

Prevalence of malnutrition and severe malnutrition diagnosed based on GLIM criteria omitting the first step or using different screening tools.

\begin{tabular}{|llllll|}
\hline Screening tool used in the first step & None & MST & MUST & NRS2002 \\
\hline \multirow{3}{*}{ GLIM criteria } & Malnutrition $(n(\%))$ & $151(26.9)$ & $115(20.5)$ & $149(26.5)$ & $79(14.1)$ \\
\cline { 2 - 6 } & Severe malnutrition $(n(\%))$ & $69(12.3)$ & $63(11.2)$ & $69(12.3)$ & $52(9.3)$ \\
\hline
\end{tabular}

GLIM, Global Leader Initiative on Malnutrition; MST, Malnutrition Screening Tool; MUST, Malnutrition Universal Screening Tool; NRS2002, Nutritional Risk Screening 2002.

Table 3 shows the variations between the classifications and the accordance between the GLIM criteria and the other screening tools (MST, MUST and NRS2002) and assessment tool (PG-SGA). MST demonstrated a better agreement $(\mathrm{k}=0.666)$ with the CLIM criteria than MUST $(\mathrm{k}=0.383)$ and NRS2002 $(\mathrm{k}=0.397)$.

Table 3

Cross tabulation of the MST, MUST, NRS2002, PG-SGA and GLIM criteria for identifying malnutrition (risk).

\begin{tabular}{|c|c|c|c|c|c|c|c|c|c|}
\hline \multirow[t]{2}{*}{ Category } & & \multicolumn{2}{|l|}{ MST } & \multicolumn{2}{|l|}{ MUST } & \multicolumn{2}{|c|}{ NRS2002 } & \multicolumn{2}{|l|}{ PG-SGA } \\
\hline & & $\begin{array}{l}\text { High } \\
\text { risk }\end{array}$ & $\begin{array}{l}\text { Low } \\
\text { risk }\end{array}$ & $\begin{array}{l}\text { Moderate/ } \\
\text { high Risk }\end{array}$ & $\begin{array}{l}\text { Low } \\
\text { risk }\end{array}$ & $\begin{array}{l}\text { High } \\
\text { risk }\end{array}$ & $\begin{array}{l}\text { Low } \\
\text { risk }\end{array}$ & Malnourished & $\begin{array}{l}\text { Well- } \\
\text { nourished }\end{array}$ \\
\hline \multirow{2}{*}{$\begin{array}{l}\text { GLIM } \\
\text { criteria }\end{array}$} & Malnourished & 115 & 36 & 149 & 2 & 79 & 72 & 144 & 7 \\
\hline & $\begin{array}{l}\text { Well- } \\
\text { nourished }\end{array}$ & 38 & 373 & 187 & 224 & 57 & 354 & 91 & 320 \\
\hline K & & 0.666 & & 0.383 & & 0.397 & & 0.623 & \\
\hline
\end{tabular}

\subsection{Agreement analysis between the GLIM criteria and PG-SGA using different screening tools in the first step}

Table 4 shows the SE, SP, PPV and NPV of GLIM criteria in diagnosing malnutrition compared with PG-SGA, in respect of omitting the first step and using MST, MUST and NRS2002 as the screening tools respectively.

GLIM framework had "fair" SE (61.3\% (95\%Cl 54.7-67.5) and "good" SP (97.9\% (95\%Cl 95.4-99.1)) when the first step was omitted, with a $\mathrm{k}$ value of 0.623 , which shows "moderate" validity in light of PG-SGA. MUST had the best performance among different screening tools applied in the first step, resulting in "fair" SE (61.3\% (95\% Cl 54.7-67.5), "good" SP $(97.9 \%(95 \% \mathrm{Cl} 95.4-99.1))$ and "moderate" validity $(\mathrm{k}=0.614)$, while MST and 
NRS2002 yielded "poor" SE (47.7\% (95\% Cl 41.2-54.2); 33.2\% (95\%Cl 27.3-39.7)) and suspicious consistency with PG-SGA ( $\mathrm{k}=0.504 ; \mathrm{K}=0.363)$.

Table 4

Sensitivity, specificity, positive and negative predictive values of GLIM framework in diagnosing malnutrition using different screening tools as the first step compared with PG-SGA.

\begin{tabular}{|c|c|c|c|c|c|}
\hline $\begin{array}{l}\text { Screening tool used in } \\
\text { the first step }\end{array}$ & $\begin{array}{l}\text { Sensitivity } \\
\%(95 \% \mathrm{Cl})\end{array}$ & $\begin{array}{l}\text { Specificity } \\
\%(95 \% \mathrm{Cl})\end{array}$ & $\begin{array}{l}\text { Positive } \\
\text { predictive value } \\
\%(95 \% \mathrm{Cl})\end{array}$ & $\begin{array}{l}\text { Negative } \\
\text { predictive value } \\
\%(95 \% \mathrm{Cl})\end{array}$ & $\mathbf{K}$ \\
\hline None & $\begin{array}{l}61.3(54.7- \\
67.5)\end{array}$ & $\begin{array}{l}97.9(95.4- \\
99.1)\end{array}$ & $95.4(90.3-98.0)$ & 77.9 (73.5-81.7) & 0.623 \\
\hline MST & $\begin{array}{l}47.7(41.2- \\
54.2)\end{array}$ & $\begin{array}{l}99.1(97.1- \\
99.8)\end{array}$ & 97.4 (92.0-99.3) & 72.5 (68.0-76.5) & 0.504 \\
\hline MUST & $\begin{array}{l}60.4(53.8- \\
66.7)\end{array}$ & $\begin{array}{l}97.9(95.4- \\
99.1)\end{array}$ & $95.3(90.2-97.9)$ & 77.5 (73.1-81.4) & 0.614 \\
\hline NRS2002 & $\begin{array}{l}33.2(27.3- \\
39.7)\end{array}$ & $\begin{array}{l}99.7(98.0- \\
100.0)\end{array}$ & 98.7 (92.2-99.9) & 67.5 (63.1-71.6) & 0.363 \\
\hline
\end{tabular}

For diagnosis of severe malnutrition, using each screening tool has similar $\mathrm{k}$ which brought into question the reliability of the GLIM criteria. Still, using MST or MUST as the screening tool or omitting the first step had "fair" SE and "good" SP, and overall "fair" validity. On the other hand, using NRS-2002 as the screening tool remained the "poor" SE, "good" SP, and overall "poor" validity of GLIM framework comparing to PG-SGA (Table 5).

Table 5

Sensitivity, specificity, positive and negative predictive values of GLIM framework in diagnosing severe malnutrition using different screening tools as the first step compared with PG-SGA.

\begin{tabular}{|c|c|c|c|c|c|}
\hline $\begin{array}{l}\text { Screening tool used in } \\
\text { the first step }\end{array}$ & $\begin{array}{l}\text { Sensitivity } \\
\%(95 \% \mathrm{Cl})\end{array}$ & $\begin{array}{l}\text { Specificity } \\
\%(95 \% \mathrm{Cl})\end{array}$ & $\begin{array}{l}\text { Positive } \\
\text { predictive value } \\
\%(95 \% \mathrm{Cl})\end{array}$ & $\begin{array}{l}\text { Negative } \\
\text { predictive value } \\
\%(95 \% \mathrm{Cl})\end{array}$ & $\mathbf{K}$ \\
\hline None & $\begin{array}{l}58.2(45.5- \\
70.0)\end{array}$ & $\begin{array}{l}93.9(91.4- \\
95.8)\end{array}$ & $56.5(44.1-68.2)$ & $94.3(91.8-96.1)$ & 0.515 \\
\hline MST & $\begin{array}{l}53.7(41.2- \\
65.8)\end{array}$ & $\begin{array}{l}94.5(92.1- \\
96.3)\end{array}$ & $57.1(44.1-69.3)$ & 93.8 (91.2-95.7) & 0.496 \\
\hline MUST & $\begin{array}{l}58.2(45.5- \\
70.0)\end{array}$ & $\begin{array}{l}93.9(91.4- \\
95.8)\end{array}$ & $56.5(44.1-68.2)$ & $94.3(91.8-96.1)$ & 0.515 \\
\hline NRS2002 & $\begin{array}{l}49.3(37.0- \\
61.6)\end{array}$ & $\begin{array}{l}96.2(94.0- \\
97.6)\end{array}$ & 63.5 (48.9-76.0) & 93.3 (90.7-95.3) & 0.503 \\
\hline $\begin{array}{l}\text { GLIM, Global Leader Ini } \\
\text { confidence interval; MS } \\
\text { NRS2002, Nutritional R }\end{array}$ & $\begin{array}{l}\text { on Malnutr } \\
\text { lutrition Sc } \\
\text { eening } 200\end{array}$ & $\begin{array}{l}\text { n; PG-SGA, } \\
\text { ning Tool; M }\end{array}$ & $\begin{array}{l}\text { ient-generated S } \\
\mathrm{T} \text {, Malnutrition } \mathrm{U}\end{array}$ & $\begin{array}{l}\text { ctive Global Ass } \\
\text { rsal Screening T }\end{array}$ & ent; Cl: \\
\hline
\end{tabular}




\section{Discussion}

Cancer patients frequently encounter nutritional and metabolic derangement, which carry prognostic significance[28,29] and is often amenable to treatment[30]. Therefore, mandatory screening and assessment are established in many clinical and health care settings, aiming to increase awareness and allow early recognition and treatment $[4,31]$. Therefore, screening and evaluation tools are expected to be brief, timesaving and efficient, especially in the outpatient setting.

Among the nutrition screening tools validated and well-used in the past decades, referring to NRS2002, MUST and MST, there is no consensus on which one is more efficient and precise in cancer patients[32]. Besides, in the newly established GLIM framework, it's suggested that any validated screening tools can be used in the first step, while which tool is better in certain population or setting is uncertain. For cancer patients, MUST[9, 21], NRS2002[33, 34] and the short form of MNA (MNA-SF)[35] have been used as the screening tool in literature, depending on the age of included patients and clinical setting. There are also studies using GLIM criteria to make nutrition assessment omitting the first step[16, 31].

As for nutrition assessment, PG-SGA is specially designed for and commonly used in cancer population, while the GLIM framework is an objective tool newly established in order to diagnose malnutrition and is initially proved to be valuable in cancer patients in predicting prognosis $[9,16,33,34]$.

Nutrition screening or nutrition risk screening aims to increase awareness of malnutrition, however, the abnormal nutrition screening results neither necessarily represent malnutrition, nor can be taken to design individualized nutrition pathways[4], with the exception that NRS2002 can be used to guide nutrition support. For patients at risk of nutrition, assessment should be done in order to establish the diagnosis of malnutrition and severity grading. The accordance between nutrition screening tools and GLIM criteria does not indicate the value as the screening tool in the first step.

In this study, we not only compared the accordance of difference screening tools with GLIM criteria, but also evaluated the performance of them in the first step of GLIM framework. Although MST $(\mathrm{K}=0.666)$ and NRS2002 $(\mathrm{K}=0.383)$ were better corelated with the GLIM criteria among ambulatory cancer patients than MUST ( $\mathrm{K}=0.383$ ), using MUST ( $\mathrm{K}=0.614$ ) as the screening tool in the first step of GLIM framework resulted in much better accordance with PG-SGA than using MST $(k=0.504)$ or NRS2002 $(\kappa=0.363)$. Hence, MUST may be the better screening tool among ambulatory cancer patients or in outpatient setting.

Still, when the first step is omitted, GLIM criteria had better performance compared to PG-SGA than using any screening tools in the first step, including the MUST. It's understandable that applying any screening tool in the first step decreased the SE as well as increased the SP compared with omitting the first step. Since GLIM criteria omitting the first step has only a "fair" SE (61.3\% (54.7-67.5)) and a high enough SP $(97.9 \%(95 \% \mathrm{Cl}$ 95.4-99.1)) in this study, the use of screening tools as the first step unsurprisingly decreased the consistency between GLIM framework and PG-SGA. Regarding the high incidence and adverse effects of malnutrition in cancer patients, along with the performance of GLIM criteria omitting first step compared to PG-SGA, it may be reasonable to assess nutritional status in every ambulatory cancer patient using GLIM criteria without nutrition screening, but whether it could bring clinical benefit to patients warrants prospective controlled clinical trials to prove. 
Unlike MST and MUST which indicate risk of malnutrition, NRS2002 represents the risk related to nutrition, predicts clinical outcome and therefor guides nutrition support[19], which have been proved by multiple retrospective and prospective studies. Xu et al. [36]reanalyzed data of a multi-center prospective cohort study, which recruited 1831 cases who received fitting requirement nutrition support therapy (support cohort) or glucose-electrolytes infusion (nonsupport cohort) respectively. Of the 827 (45.2\%) cases who were NRS2002 positive, 391 (21.4\%) cases were identified as malnourished by GLIM criteria. For the other 436 (23.8\%) cases who were NRS2002 positive but GLIM negative, the rate of infection was significantly lower in the support cohort than in the nonsupport cohort $(8.0 \%$ vs. $15.7 \%$; $=0.011)$, which indicated that GLIM criteria neglected half of the patients who could benefit from nutritional support by decreasing the rate of infection complications[36]. On the other hand, would patients who are NRS2002 negative but GLIM positive benefit from nutritional support? Part of patients with a NRS2002 score $\leq 3$ may also gain positive clinical effect from nutrition support, though the proportion of patients who might benefit decreased as the score decreased[19]. Will GLIM positive represent the part of the NRS200 negative patients who will benefit from nutritional support? In our study, NRS2002 negative and GLIM criteria positive patients took up $12.8 \%$ of participants, which makes the question warrant to answer. However, up to now, there is no literature answering this question, since NRS2002 is commonly used as the indicator of nutritional intervention and researchers usually chose NRS2002 as the screening tool in the first step of GLIM criteria[33,34]. Real world study may help up to answer the question initially.

As discussed above, we recommend routine screening of nutrition risk by NRS2002, and routine nutrition evaluation by GLIM framework omitting the screening step, if it were not for workload. When conditions do not warrant routine nutritional assessment, MUST could be chosen as the screening tool in the first step of GLIM framework for the sake of decreasing evaluating burden as well as maintaining accuracy.

Our study has some strengths. As a prospective cross-sectional observational study, we recruited 562 patients in a short period of time, namely 3 weeks, and all data for MST, MUST, NRS2002, PG-SGA and GLIM were collected concurrently, which ensured that no data was omitted, and the conclusion drawn from the study is more reliable than retrospective study. Our study not only assessed the accordance of between different nutrition screening tools and GLIM criteria, but also evaluated the diagnostic capacity of them as the first step of GLIM framework. It's recommended that any validated nutrition screening tools can be used in the first step of GLIM framework, but, to our knowledge, there have been no published study focusing on the best choice of screening tools. Moreover, body composition information such as FFMI or ASMI was usually unavailable in published articles and considered as important and warranted in validation of GLIM criteria[26]. In the current study, we applied BIA in every participant and provided relatively precise evaluation of reduced muscle mass. As shown in our recently published study focusing on interrater reliability of the GLIM criteria using the same data, the appliance of body composition measurement improved the performance of GLIM framework compared to PG-SGA[21].

All the same, there are several limitations in our study. Since it was a single-center observational study, results should be interpreted with caution. Food intake reduction was assessed via self-report on general amount of food intake instead of an in-depth diet history for sake of promoting feasibility, thus the accuracy of food intake evaluation was impaired. Inflammation was assessed by increased CRP and current infection in this study. However, the detection rate of CRP was low in our study, while no patients with current infectious disease was 
admitted in our day oncology unit, so the evaluation of inflammation status is inadequate, which could lead to certain bias. Moreover, though recommended by a recent review, PG-SGA as the gold standard to validate the GLIM criteria is not well-accepted by all clinician and nutritionist. Comprehensive evaluation or clinical outcome such as survival situation or complications rates could be more valuable in the validation of GLIM criteria. We need to follow up the participants to collect survival data and information of adverse complications in order to reveal the value of GLIM criteria in predicting clinical outcomes and guiding nutrition support.

\section{Conclusions}

In general, our study indicates that GLIM framework omitting the first step has best validity comparing with PGSGA in ambulatory setting. Since cancer patients are at high risk of malnutrition, it's reasonable and more favorable to assess nutritional status routinely regardless of the result of screening. When conditions do not warrant routine nutritional assessment, MUST might be the most suitable screening tool as the first step of GLIM framework for ambulatory cancer patients.

\section{Declarations}

Funding: This research was funded by the Beijing Municipal Administration of Hospitals' Mission Plan (to Wei LIU), grant number SML20181102, the Leading Talents of Science and Technology Innovation in the National "Ten thousand Talents Program" (to Wei LIU), and the Science Foundation of Peking University Cancer Hospital (to Yanfei Wang), grant number 2021-23.

Conflicts of interest/Competing interests: The authors declare no conflict of interest.

Availability of data and material: The data related to the article have not been disclosed at present because the data processing and use have not been completely completed.

Code availability: Not applicable.

Authors' contributions: Conceptualization, Y.W. (Yanfei Wang), Z. L. (Ziqi Liu), Y.F., Z.P., and W.L.; methodology, Y.W. (Yanfei Wang), Z. L. (Ziqi Liu); software, Y.W. (Yanfei Wang) ; validation, X.C., Y.W. (Yunyi Wang); formal analysis, Y.W. (Yanfei Wang), Z. L. (Ziqi Liu),X.C., Y.W. (Yunyi Wang); investigation, Y.W. (Yanfei Wang), Z. L. (Ziqi Liu),X.C., Y.W. (Yunyi Wang); data curation, Y.W. (Yanfei Wang), Z.L.(Ziqi Liu), X.C., Y.W. (Yunyi Wang), Z. L. (Zhongfen Liu), Y.Z., D.J., L.X,; writing-original draft preparation, Y.W. (Yanfei Wang), Z.L.(Ziqi Liu); writingreview and editing, X.C.; Y.W. (Yunyi Wang); Z.L.(Zhongfen Liu), Y.Z., D.J., L.X., Y.F., Z.P., and W.L.; project administration, Y.F., Z.P., and W.L.; funding acquisition, W.L. and Y.W. (Yanfei Wang). All authors have read and agreed to the published version of the manuscript.

Ethics approval: The study was conducted according to the guidelines of the Declaration of Helsinki and approved by the independent institutional ethics committee of the Peking University Cancer Hospital.

Consent to participate: Informed consent was obtained from all subjects involved in the study.

Consent for publication: Not applicable. 


\section{References}

1. Hebuterne, X., et al., Prevalence of malnutrition and current use of nutrition support in patients with cancer. JPEN J Parenter Enteral Nutr, 2014. 38(2): p. 196-204.

2. Arends, J., et al., ESPEN expert group recommendations for action against cancer-related malnutrition. Clin Nutr, 2017. 36(5): p. 1187-1196.

3. Salas, S., et al., Nutritional factors as predictors of response to radio-chemotherapy and survival in unresectable squamous head and neck carcinoma. Radiother Oncol, 2008. 87(2): p. 195-200.

4. Arends, J., et al., ESPEN guidelines on nutrition in cancer patients. Clin Nutr, 2017. 36(1): p. 11-48.

5. Hill, A., et al., Associations between nutritional status, weight loss, radiotherapy treatment toxicity and treatment outcomes in gastrointestinal cancer patients. Clin Nutr, 2011. 30(1): p. 92-8.

6. Tian, J. and J.S. Chen, Nutritional status and quality of life of the gastric cancer patients in Changle County of China. World J Gastroenterol, 2005. 11(11): p. 1582-6.

7. Planas, M., et al., Prevalence of hospital malnutrition in cancer patients: a sub-analysis of the PREDyCES(R) study. Support Care Cancer, 2016. 24(1): p. 429-435.

8. Rondel, A., et al., The new ESPEN diagnostic criteria for malnutrition predict overall survival in hospitalised patients. Clin Nutr, 2018. 37(1): p. 163-168.

9. Contreras-Bolivar, V., et al., GLIM Criteria Using Hand Grip Strength Adequately Predict Six-Month Mortality in Cancer Inpatients. Nutrients, 2019. 11(9).

10. Lin, J., et al., Severe weight loss during preoperative chemoradiotherapy compromises survival outcome for patients with locally advanced rectal cancer. J Cancer Res Clin Oncol, 2016. 142(12): p. 2551-2560.

11. Jensen, G.L., et al., GLIM Criteria for the Diagnosis of Malnutrition: A Consensus Report From the Global Clinical Nutrition Community. JPEN J Parenter Enteral Nutr, 2019. 43(1): p. 32-40.

12. Cederholm, T., et al., GLIM criteria for the diagnosis of malnutrition - A consensus report from the global clinical nutrition community. Clin Nutr, 2019. 38(1): p. 1-9.

13. Keller, H., et al., Global Leadership Initiative on Malnutrition (GLIM): Guidance on Validation of the Operational Criteria for the Diagnosis of Protein-Energy Malnutrition in Adults. JPEN J Parenter Enteral Nutr, 2020. 44(6): p. 992-1003.

14. de van der Schueren, M.A.E., et al., Global Leadership Initiative on Malnutrition (GLIM): Guidance on validation of the operational criteria for the diagnosis of protein-energy malnutrition in adults. Clin Nutr, 2020. 39(9): p. 2872-2880.

15. Ottery, F.D., Definition of standardized nutritional assessment and interventional pathways in oncology. Nutrition, 1996. 12(1 Suppl): p. S15-9.

16. De Groot, L.M., et al., Malnutrition Screening and Assessment in the Cancer Care Ambulatory Setting: Mortality Predictability and Validity of the Patient-Generated Subjective Global Assessment Short form (PGSGA SF) and the GLIM Criteria. Nutrients, 2020. 12(8).

17. Ferguson, M., et al., Development of a valid and reliable malnutrition screening tool for adult acute hospital patients. Nutrition, 1999. 15(6): p. 458-64.

18. Stratton, R.J., et al., Malnutrition in hospital outpatients and inpatients: prevalence, concurrent validity and ease of use of the 'malnutrition universal screening tool' ('MUST') for adults. Br J Nutr, 2004. 92(5): p. 799- 
808.

19. Kondrup, J., et al., Nutritional risk screening (NRS 2002): a new method based on an analysis of controlled clinical trials. Clin Nutr, 2003. 22(3): p. 321-36.

20. Shi, H., W. Li, and Y. Qi, Nutrition screening and evaluation. 2014, Beijing: People's Medical Publishing House.

21. Wang, Y., et al., Body Composition Measurement Improved Performance of GLIM Criteria in Diagnosing Malnutrition Compared to PG-SGA in Ambulatory Cancer Patients: A Prospective Cross-Sectional Study. Nutrients, 2021. 13(8).

22. Chen, L.K., et al., Asian Working Group for Sarcopenia: 2019 Consensus Update on Sarcopenia Diagnosis and Treatment. J Am Med Dir Assoc, 2020. 21(3): p. 300-307 e2.

23. Cederholm, T., et al., ESPEN guidelines on definitions and terminology of clinical nutrition. Clin Nutr, 2017. 36(1): p. 49-64.

24. Maeda, K., et al., Reference body mass index values and the prevalence of malnutrition according to the Global Leadership Initiative on Malnutrition criteria. Clin Nutr, 2020. 39(1): p. 180-184.

25. Shrout, P.E., Measurement reliability and agreement in psychiatry. Stat Methods Med Res, 1998. 7(3): p. 301-17.

26. Allard, J.P., et al., GLIM criteria has fair sensitivity and specificity for diagnosing malnutrition when using SGA as comparator. Clin Nutr, 2020. 39(9): p. 2771-2777.

27. Power, L., et al., A review of the validity of malnutrition screening tools used in older adults in community and healthcare settings - A MaNuEL study. Clin Nutr ESPEN, 2018. 24: p. 1-13.

28. McMillan, D.C., The systemic inflammation-based Glasgow Prognostic Score: a decade of experience in patients with cancer. Cancer Treat Rev, 2013. 39(5): p. 534-40.

29. Martin, L., et al., Diagnostic criteria for the classification of cancer-associated weight loss. J Clin Oncol, 2015. 33(1): p. 90-9.

30. Pan, H., et al., The impact of nutritional status, nutritional risk, and nutritional treatment on clinical outcome of 2248 hospitalized cancer patients: a multi-center, prospective cohort study in Chinese teaching hospitals. Nutr Cancer, 2013. 65(1): p. 62-70.

31. Zhang, Z., et al., Prevalence of malnutrition comparing NRS2002, MUST, and PG-SGA with the GLIM criteria in adults with cancer: A multi-center study. Nutrition, 2021. 83: p. 111072.

32. Thompson, K.L., et al., Oncology Evidence-Based Nutrition Practice Guideline for Adults. J Acad Nutr Diet, 2017. 117(2): p. 297-310 e47.

33. Zhang, X., et al., The GLIM criteria as an effective tool for nutrition assessment and survival prediction in older adult cancer patients. Clin Nutr, 2021. 40(3): p. 1224-1232.

34. Yin, L., et al., Is hand grip strength a necessary supportive index in the phenotypic criteria of the GLIMbased diagnosis of malnutrition in patients with cancer? Support Care Cancer, 2021.

35. Sobrini, P., C. Sanchez-Castellano, and A.J. Cruz-Jentoft, MNA-SF as a screening tool for malnutrition diagnosed with the glim criteria in older persons with cancer. Eur Geriatr Med, 2021.

36. Xu, J.Y., et al., Nutritional support therapy after GL IM criteria may neglect the benefit of reducing infection complications compared with NRS2002: Reanalysis of a cohort study. Nutrition, 2020. 79-80: p. 110802. 
Figures

\section{$\mathrm{N}=\mathbf{6 8 6}$}

Patients admitted to day oncology unit, Peking University Cancer Hospital, from 11

November 2020 to 10 December 2020

$$
\mathbf{N}=\mathbf{9 2}
$$

Patients failed inclusion criteria

(1) Under 18 years old $\mathrm{N}=3$

(2) Not receiving intravenous treatment $\mathrm{N}=89$

Figure 1

Flow chart.

\section{$\mathbf{N}=32$}

Patients fit exclusion criteria

(1) In poor performance status $\mathrm{N}=3$

(2) Unable to stand up due to illness $N=1$

(3) Unwilling to participate in this study $\mathrm{N}=28$

(3) Receiving in-chair intravenous treatment

(4) Having normal cognitive function

$$
\mathrm{N}=562
$$ \\ Patients included in this study}

